\title{
Rolling-shutter-based 16- QAM optical camera communication by spatial luminance distribution
}

\author{
Wataru Chujo ${ }^{\text {a) }}$ and Masahiro Kinoshita \\ Department of Electrical and Electronic Engineering, Meijo University, \\ 1-501 Shiogamaguchi, Tempaku-ku, Nagoya 468-8502, Japan \\ a)wchujo@meijo-u.ac.jp
}

\begin{abstract}
To increase both the symbol rate and the number of bits per symbol for optical camera communication (OCC), rolling-shutter (RS)-based quadrature amplitude modulation (QAM) is demonstrated by spatial luminance distribution of a light emitting diode (LED) surface. The spatial luminance distribution method is adapted to different LEDs regardless of the LED shape and configuration, and compared with the conventional pixel value method. The advantages and drawbacks of the spatial luminance distribution method are experimentally investigated. When the symbol length is integer multiples of the line interval of the RS camera, RS-based 16-QAM OCC is demonstrated using a square-shape LED.
\end{abstract}

Keywords: visible light communication, optical camera communication, image sensor, rolling shutter, QAM, spatial luminance distribution

Classification: Wireless Communication Technologies

\section{References}

[1] C. Danakis, M. Afgani, G. Povey, I. Underwood, and H. Haas, "Using a CMOS camera sensor for visible light communication," IEEE Globecom Workshops, Anaheim, USA, pp. 1244-1248, Dec. 2012. DOI:10.1109/GLOCOMW.2012. 6477759

[2] H. Aoyama and M. Oshima, "Line scan sampling for visible light communication: Theory and practice," IEEE International Conference on Communications, London, UK, pp. 5060-5065, June 2015. DOI:10.1109/ICC. 2015.7249126

[3] P. Luo, M. Zhang, Z. Ghassemlooy, H. L. Minh, H.-M. Tsai, X. Tang, and D. Han, "Experimental demonstration of a 1024-QAM optical camera communication system," IEEE Photon. Technol. Lett., vol. 28, no. 2, pp. 139-142, Jan. 2016. DOI:10.1109/LPT.2015.2487544

[4] V. P. Rachim and W.-Y. Chung, "Multilevel intensity-modulation for rolling shutter-based optical camera communication," IEEE Photon. Technol. Lett., vol. 30, no. 10, pp. 903-906, Apr. 2018. DOI:10.1109/LPT.2018.2823784

[5] W. Chujo and M. Kinoshita, "Rolling-shutter-based QPSK by spatial luminance distribution for optical camera communication," 2018 IEEE Globecom Workshops, Abu Dhabi, UAE, Dec. 2018. DOI:10.1109/GLOCOMW.2018.8644404 
[6] J. M. Kahn and J. R. Barry, "Wireless infrared communications," Proc. IEEE, vol. 85, no. 2, pp. 265-298, Feb. 1997. DOI:10.1109/5.554222

\section{Introduction}

OCC using an LED lamp and smartphone's built-in camera is effective for indoor internet of things applications. However, to increase the maximum data rate available for OCC, increases in both the symbol rate and the number of bits per symbol are necessary. Since RS-based OCC is effective to increase the symbol rate using the LED lamp [1, 2], RS-based multilevel-QAM OCC is important to increase the number of bits per symbol further.

As a multilevel phase and amplitude modulation, 1024QAM has been experimentally demonstrated with under-sampled subcarrier modulation [3]. However, RS-based OCC has not been conducted. As a multilevel amplitude modulation for RS-based OCC, multilevel data symbol is constructed from multiple pulses in a single symbol period using RGB-LED [4]. However, RS-based multilevel QAM OCC has not been conducted. In addition, pixel value of the captured LED image is used for amplitude demodulation.

By contrast, we propose RS-based multilevel-QAM OCC by the spatial luminance distribution method. The method generates multilevel QAM symbols from a single pulse in a single symbol period using a white LED. We have experimentally demonstrated 256QAM by spatial luminance distribution of a two-dimensional LED image [5]. Moreover, RS-based 4-QAM OCC has been demonstrated by the method using a round-shape LED and off-the-shelf RS camera [5]. In this study, RS-based multilevel-QAM OCC is demonstrated by the method using different kind of LEDs. The method is also compared with the conventional pixel value method, and advantages and drawbacks of the method are shown to verify effectiveness of the spatial luminance distribution method.

\section{Principle of spatial luminance distribution method}

Pixel value is generally used to know the luminance of the captured image such as LED display or LED traffic signal because the luminance of those LEDs is not much higher than ambient luminance. However, luminance of the LED lamp is always much higher than the ambient luminance. Pixel values on the image of a white LED lamp are constantly saturated in the maximum level, even when the exposure value is set at minimum value. Therefore, the spatial luminance distribution method is necessary for OCC using the LED lamp as a transmitter.

Fig. 1(a) shows relationship between LED luminance and LED image captured with RS camera which corresponds to the spatial luminance distribution of the LED screen surface. A white LED lamp consists of an LED source and a screen surface. The spatial luminance distribution on the screen surface is determined based on the Lambertian model [6]. Since the luminance is proportional to $\cos \psi$, where $\psi$ is the angle between the screen surface normal and the direction of the incoming light, the luminance on the screen surface gradually decreases toward the end of the surface. 
Therefore, when the LED luminance is changed in the form of a sine wave, spatial luminance distribution of the screen surface is also changed in the form of a sine wave. Since the RS camera captures the spatial luminance distribution as an image on the screen at the line intervals of time, number of white pixels on each line of the screen is also changed in the form of a sine wave. LED transmitter's symbol length is selected to be integer multiples of the line interval of RS camera. Number of white pixels on each line of the screen is counted.

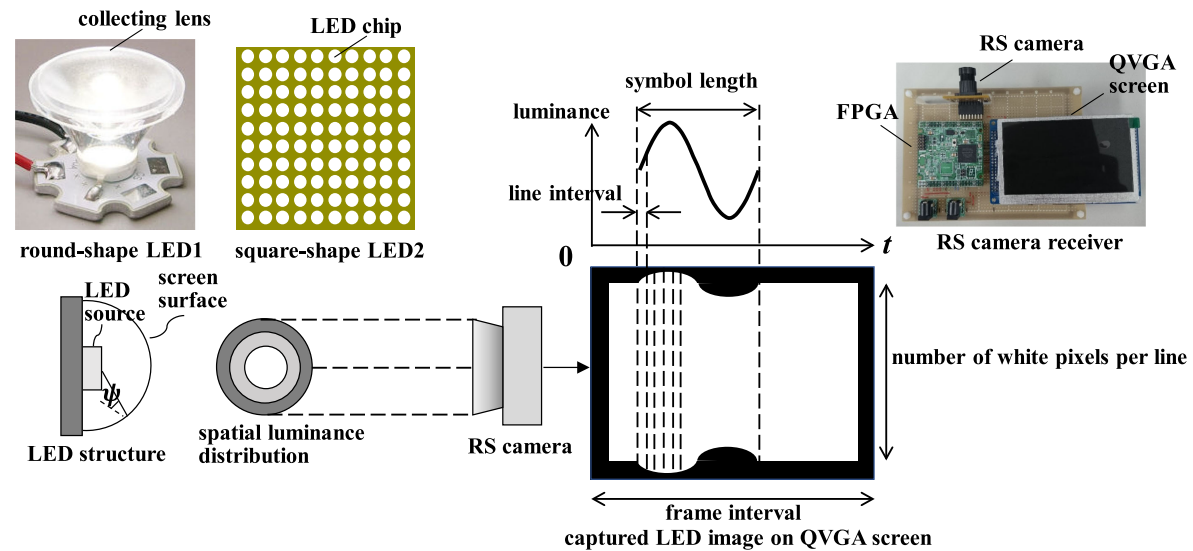

(a) relationship between LED luminance and captured LED image

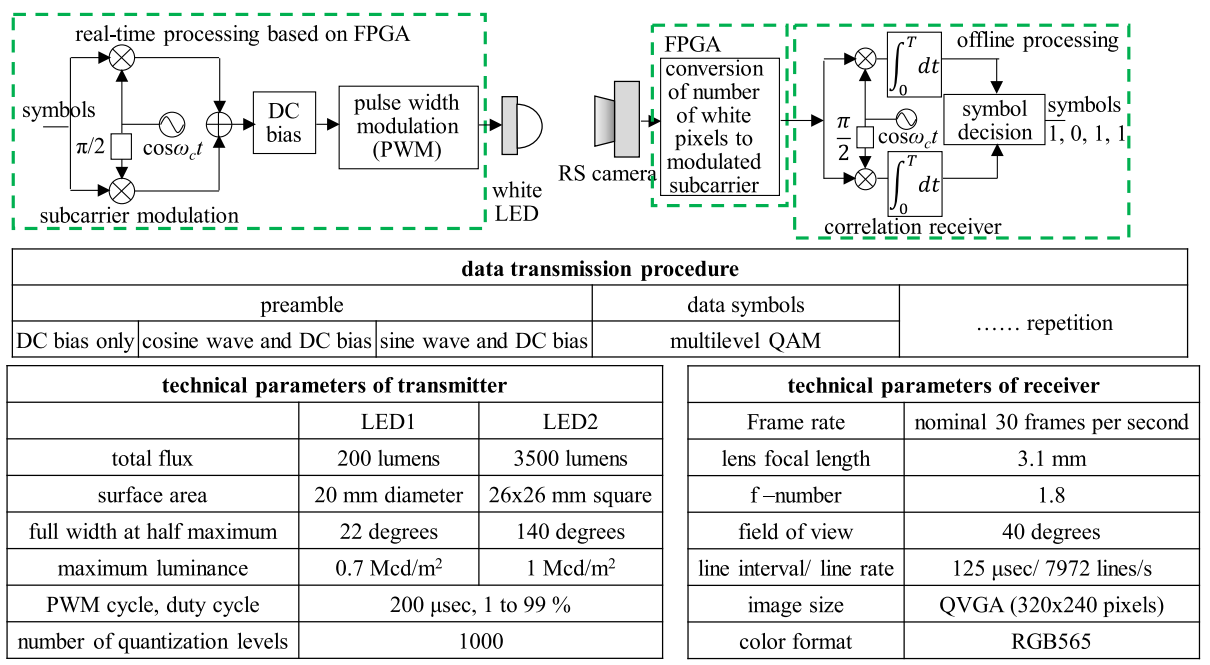

(b) configuration and technical parameters of LED transmitter and RS camera receiver

Fig. 1. Principle of spatial luminance distribution method.

Fig. 1(b) shows configuration and technical parameters of the LED transmitter and RS camera receiver. In the LED transmitter, cosine- and sine-wave subcarriers are modulated with symbols and both are combined. Next, DC bias is added. Finally, LED luminance is changed with pulse width modulation (PWM) in accordance with the modulated subcarrier.

Transmitted symbols consist of preamble and data symbols in data transmission procedure as shown in Fig. 1(b). The preamble consists of three kinds of symbols. First, only DC bias is transmitted to know the symbol timing. Next, cosineand sine-wave subcarriers are transmitted as reference symbols to correlate with 
the following data symbols in the receiver. Next, multilevel-QAM data symbols are transmitted. Two kinds of LEDs are used to demonstrate the spatial luminance distribution method regardless of the LED shape or configuration. LED1 is a single round-shape LED with a $20 \mathrm{~mm}$ diameter collecting lens. LED2 is $25 \times 25 \mathrm{~mm}$ square-shape LED which consists of $10 \times 10$ small LED chips.

On the other hand, in RS camera receiver, number of white pixels on each line of the captured LED image is counted by setting a threshold at pixel values and converted to the modulated subcarrier. Maximum likelihood detection is conducted with a correlation receiver to recover the symbols.

\section{Constellation diagrams of RS-based multilevel-QAM OCC}

RS-based multilevel-QAM OCCs are demonstrated using white light LED transmitters and off-the-shelf RS camera receivers by the spatial luminance distribution method. Since exposure time of the RS camera is not independently controlled, 8-bit exposure value is set at minimum value, " 0 " to minimize the exposure time. However, the minimum exposure time seems to be still much longer than the line interval of the RS camera. Multilevel QAM is conducted by decreasing the symbol rate to be integer division of the line rate of the RS camera.

Constellation diagrams of 4QAM were measured at different symbol rates under sufficient ambient lighting. Measured distance between transmitters and a receiver is 20 and 17 centimeters using LED1 and LED2, respectively. The threshold of pixel values is set at maximum value, "11111 11111111111 ," in RGB565 color format to discriminate white pixels. Fig. 2(a) shows measured 4-QAM constellation diagram versus symbol rate using the round-shape LED1. Transmitter's symbol rate was decreased to $1 / 6$ and $1 / 8$ of the line rate of the RS camera. Both constellation points are within the thresholds on 4QAM. However, it is difficult to decrease the symbol rate further because the number of pixels on each line gradually decreases towards the edge of the LED due to the round shape.

On the other hand, Fig. 2(b) shows measured 4-QAM constellation diagram versus symbol rate using the square-shape LED2. Symbol rate was decreased to $1 / 6,1 / 8,1 / 12$ and $1 / 20$ of the line rate of the RS camera. Although measured constellation points vary, the phase and amplitude inaccuracies of the square-shape LED2 are less than those of the round-shape LED1. Since the number of pixels on each line of the square-shape LED2 image is almost constant compared with that of the round-shape LED1 image, the phase and amplitude accuracies of each constellation point are improved. Moreover, as the symbol rate decreases, phase and amplitude accuracies are gradually improved. According to these results, the long exposure time of off-the-shelf RS camera seems to affect the performance of 4-QAM constellations.

Next, to clarify the advantages and drawbacks of the proposed method, the method was compared with the conventional pixel value method. Fig. 2(c) shows measured 4-QAM constellation diagram by the pixel value method. LED luminance needs to be carefully selected not to saturate the pixel value of the RS camera. Although measured constellation points are stable at approximately 65 $\mathrm{kcd} / \mathrm{m}^{2}$, the accuracy of constellation points degrades at approximately $100 \mathrm{kcd} / \mathrm{m}^{2}$ 
by the saturation of the pixel values. When the luminance is more than $100 \mathrm{kcd} / \mathrm{m}^{2}$, it was impossible to measure the constellation diagram. On the other hand, the accuracy of constellation points degrades further at approximately 15 and 1.5 $\mathrm{kcd} / \mathrm{m}^{2}$ due to decrease of the pixel values and influence of the background light. Since the suitable luminance needs to be carefully selected, the conventional pixel value method is not suitable for OCC using the LED lamp.

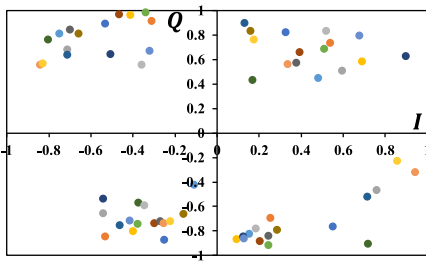

ratio of symbol rate to line rate is $1 / 6$ (2.657 kbps)

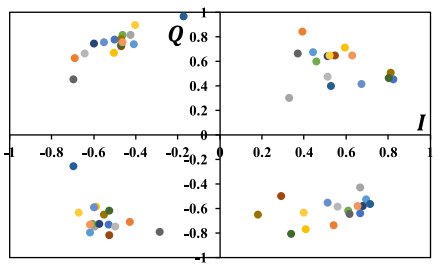

ratio of symbol rate to line rate is $1 / 8$ (1.993 kbps)

(a) spatial luminance distribution method using the round-shape LED1

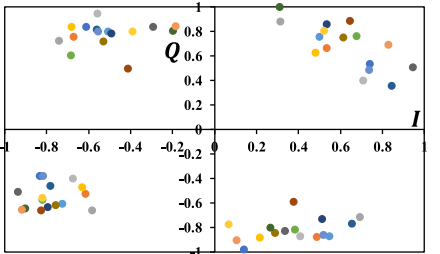

ratio of symbol rate to line rate is $1 / 6$ (2.657 kbps)

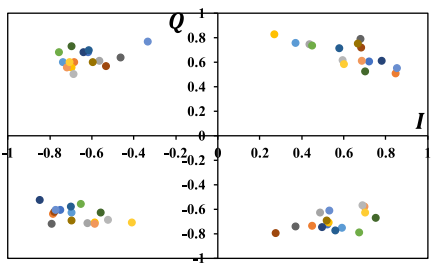

ratio of symbol rate to line rate is $1 / 12$ (1.329 kbps)

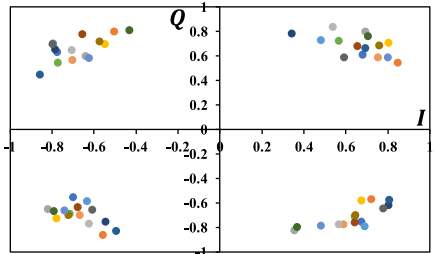

ratio of symbol rate to line rate is $1 / 8$ (1.993 kbps)

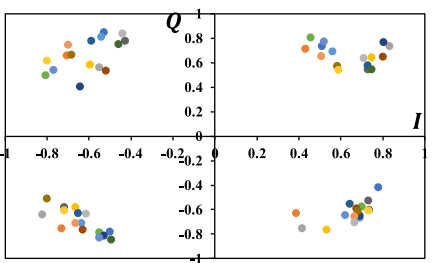

ratio of symbol rate to line rate is $1 / 20$ (797 bps)

(b) spatial luminance distribution method using the square-shape LED2

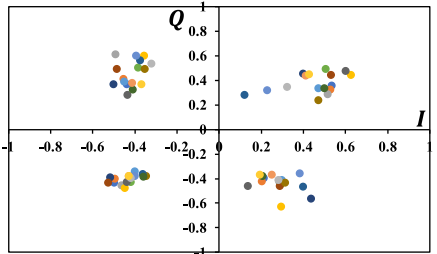

luminance is approximately $100 \mathrm{kcd} / \mathrm{m}^{2}$

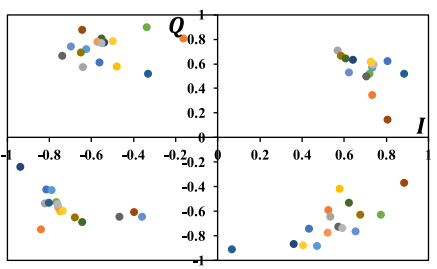

luminance is approximately $15 \mathrm{kcd} / \mathrm{m}^{2}$

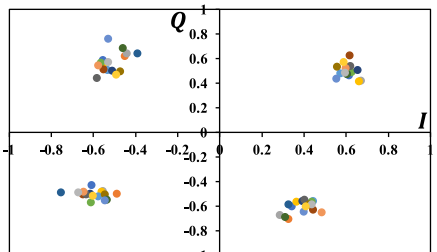

luminance is approximately $65 \mathrm{kcd} / \mathrm{m}^{2}$

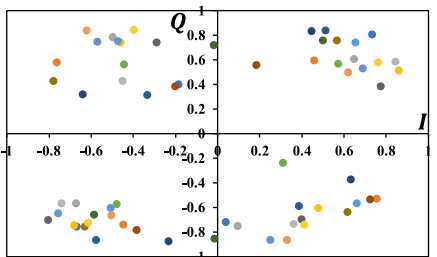

luminance is approximately $1.5 \mathrm{kcd} / \mathrm{m}^{2}$

(c) conventional pixel value method using the square-shape LED2, where the ratio of symbol rate to line rate is $1 / 8(1.993 \mathrm{kbps})$.

Fig. 2. Measured 4-QAM constellation diagrams. 
Furthermore, to clarify the advantages and drawbacks of the proposed method, communication range was evaluated. Fig. 3(a) shows measured 4-QAM constellation diagram versus distance, where 6-bit $\mathrm{G}$ value in RGB565 color format is used as the threshold value. As the distance increases, the threshold $G$ value was decreased to maintain the captured image size sufficiently. Even when the distance increases, 4QAM was achieved by decreasing the threshold $\mathrm{G}$ value. However, the accuracy of constellation points gradually degrades at $100 \mathrm{~cm}$ due to decrease of the threshold $G$ value and influence of the background light.

Finally, Fig. 3(b) shows measured 16-QAM constellation diagram using the square-shape LED2, where the symbol rate is decreased to be $1 / 12$ and $1 / 16$ of the line rate of the RS camera. Although measured constellation points still vary, all the constellation points are within the thresholds on 16QAM. RS-based 16-QAM OCC was experimentally demonstrated to increase the number of bits per symbol.

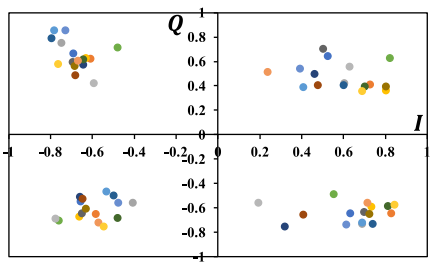

distance is $45 \mathrm{~cm}$, and $\mathrm{G}$ value is " 100000 "

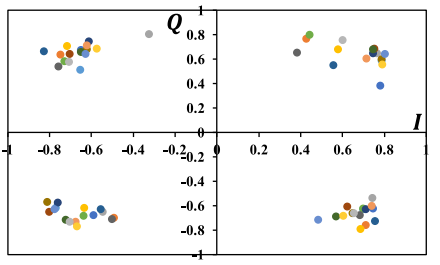

distance is $75 \mathrm{~cm}$, and $G$ value is " 001100 " distance is $100 \mathrm{~cm}$, and $G$ value is " 001100 "

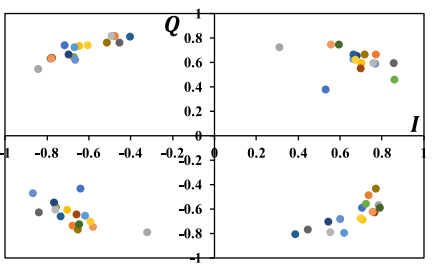

distance is $60 \mathrm{~cm}$, and $\mathrm{G}$ value is " 010000 "

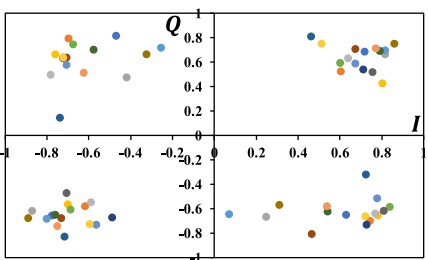

(a) measured 4-QAM constellation diagram versus distance, where the ratio of symbol rate to line rate is $1 / 8(1.993 \mathrm{kbps})$.

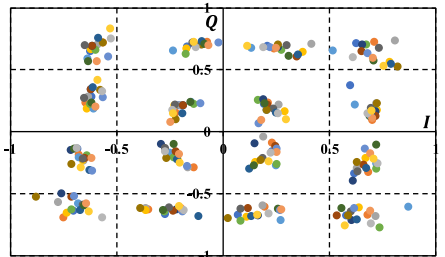

ratio of symbol rate to line rate is $1 / 12$ $(2.657 \mathrm{kbps})$

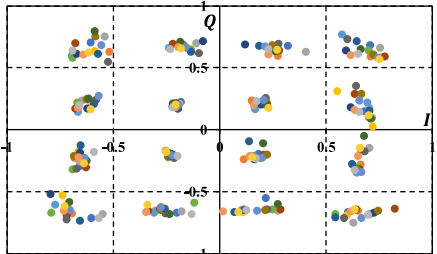

ratio of symbol rate to line rate is $1 / 16$ (1.993 bps)

(b) measured 16-QAM constellation diagram versus symbol rate

Fig. 3. Measured 4-QAM and 16-QAM constellation diagrams.

\section{Conclusion}

To increase both the symbol rate and the number of bits per symbol for OCC, RSbased QAM was demonstrated by the spatial luminance distribution method. The method was adapted to round- and square-shape LEDs and compared with the conventional pixel value method to clarify the advantages and drawbacks of the method. RS-based 16-QAM OCC was achieved when the symbol rate is $1 / 12$ and $1 / 16$ of the line rate of RS camera to increase the number of bits per symbol. To increase the data rate further, RS camera with short exposure time is necessary. 